\title{
Observation of Hydrogen-Induced Dzyaloshinskii-Moriya Interaction and Reversible Switching of Magnetic Chirality
}

\author{
Gong Chen $\odot,{ }^{1,2, *}$ MacCallum Robertson $\odot,{ }^{1}$ Markus Hoffmann $\odot,{ }^{3}$ Colin Ophus $\odot,{ }^{4}$ André L. Fernandes Cauduro $\odot,{ }^{4}$ \\ Roberto Lo Conte $\odot,{ }^{5,6}$ Haifeng Ding $\odot,{ }^{7}$ Roland Wiesendanger $\odot,{ }^{6}$ Stefan Blügel $\odot,{ }^{3}$ \\ Andreas K. Schmid $\odot,{ }^{4}$ and Kai Liu $\odot^{1,2, \dagger}$ \\ ${ }^{1}$ Physics Department, University of California, Davis, California 95616, USA \\ ${ }^{2}$ Physics Department, Georgetown University, Washington, DC 20057, USA \\ ${ }^{3}$ Peter Grünberg Institut and Institute for Advanced Simulation, \\ Forschungszentrum Jülich and JARA, 52425 Jülich, Germany \\ ${ }^{4}$ NCEM, Molecular Foundry, Lawrence Berkeley National Laboratory, Berkeley, California, 94720, USA \\ ${ }^{5}$ Department of Materials Science \& Engineering, \\ University of California, Berkeley, California 95720, USA \\ ${ }^{6}$ Department of Physics, University of Hamburg, D-20355 Hamburg, Germany \\ ${ }^{7}$ National Laboratory of Solid State Microstructures, Department of Physics and Collaborative Innovation \\ Center of Advanced Microstructures, Nanjing University, Nanjing 210093, People's Republic of China
}

(Received 29 July 2020; revised 15 January 2021; accepted 1 March 2021; published 16 April 2021)

\begin{abstract}
The Dzyaloshinskii-Moriya interaction (DMI) has drawn much attention, as it stabilizes magnetic chirality, with important implications in fundamental and applied research. This antisymmetric exchange interaction is induced by the broken inversion symmetry at interfaces or in noncentrosymmetric lattices. Significant interfacial DMIs are often found at magnetic/heavy-metal interfaces with large spin-orbit coupling. Recent studies have shown promise for induced DMI at interfaces involving light elements such as carbon (graphene) or oxygen. Here, we report direct observation of induced DMI by chemisorption of the lightest element, hydrogen, on a ferromagnetic layer at room temperature, which is supported by density functional theory calculations. We further demonstrate a reversible chirality transition of the magnetic domain walls due to the induced DMI via hydrogen chemisorption and desorption. These results shed new light on the understanding of DMI in low atomic number materials and the design of novel chiral spintronics and magneto-ionic devices.
\end{abstract}

DOI: $10.1103 /$ PhysRevX.11.021015

Subject Areas: Condensed Matter Physics,

Magnetism, Spintronics

\section{INTRODUCTION}

The Dzyaloshinskii-Moriya interaction (DMI) is an antisymmetric exchange interaction, which can be induced in systems with broken inversion symmetry [1,2], such as the initially proposed $\mathrm{Fe}_{2} \mathrm{O}_{3}$ with weak ferrimagnetism, bulk materials with broken inversion symmetry like B20 compounds [3,4], or thin film systems [5,6]. The presence of the DMI may introduce various types of chiral magnetic structures, including helical spin spirals [3], helical or

\footnotetext{
*To whom correspondence should be addressed. gchenncem@gmail.com

${ }^{\dagger}$ To whom correspondence should be addressed. Kai.Liu@georgetown.edu

Published by the American Physical Society under the terms of the Creative Commons Attribution 4.0 International license. Further distribution of this work must maintain attribution to the author(s) and the published article's title, journal citation, and DOI.
}

Bloch-type skyrmions [4,7], and magnetic bobbers [8] in bulk materials, or cycloidal spirals [5,9], Néel-type skyrmions [6,10-12], and Néel-type chiral domain walls [13-16] in thin films. The nonzero topological charge in chiral magnetic structures adds an exciting degree of freedom [7,17], which is fundamentally intriguing. It enables novel chiral spintronic applications with ultralow-energy consumption, e.g., racetrack memories or neuromorphic computing devices [10-12,18-21], where the ability to sensitively control the chirality, stability, or size of the spin texture is critical. For instance, in the limit of stable skrymions, the radius of the skyrmion may be tailored by orders of magnitude with a fine-tuning of the DMI strength [22]. Considering that many response quantities, e.g., magnetoresistive readout, scale with the area of the skyrmion, controlling the DMI on a fine scale has important technological ramifications.

In thin films, it has been found experimentally that significant interfacial DMIs can be induced when magnetic layers are adjacent to transition metals of large spin-orbit 
interactions [10-12], oxides [23,24], or light elements such as graphene [25,26] or oxygen [27]. Theoretical calculations based on the Fert-Levy model [28] reveal that the transition-metal-induced DMI originates from the strong spin-orbit coupling combined with the wave function or charge asymmetry, which can be measured by the electric dipole moment [29]. The magnitude of the DMI is closely related to the band filling and hybridization between $5 d$ and $3 d$ orbitals near the Fermi level [30]. On the other hand, the light-element-induced DMI may be explained by the Rashba effect [25] and the charge asymmetry resulting from the charge transfer and hybridization of the band structure at the interface [31].

In this context, manipulating surfaces and interfaces with the lightest element, hydrogen, is potentially a powerful path to control the DMI. Hydrogen absorption has been shown to significantly alter properties of magnetic materials, which permits tuning of interlayer exchange coupling [32], tailoring of magnetic anisotropy [33,34], or realization of magnetoelectric coupling in antiferromagnetic oxides [35]. Hsu et al. observed a hydrogenation-assisted formation of skyrmions in the $\mathrm{Fe} / \operatorname{Ir}(111)$ system at $4.2 \mathrm{~K}$, which is attributed to the modification of the Heisenberg exchange and DMI based on $a b$ initio calculations [36]. A recent theory also predicts that hydrogen absorption on graphene alters the DMI at the graphene/Co interface [37].

On the other hand, few studies have distinguished hydrogen-chemisorption-induced effects on magnetic thin films, i.e., adsorption on the surface without penetrating the film. There have been reports on hydrogen-adsorptioninduced effects on magnetic anisotropy [38,39]. However, direct and quantitative experimental confirmation of hydrogen-induced DMI is still lacking. Furthermore, Tan et al. have demonstrated $\mathrm{H}^{+}$-based reversible magnetoionic switching at room temperature, where electric-fieldcontrolled hydrogen transport to the buried $\mathrm{Co} / \mathrm{GdO}$ interface is used to toggle the perpendicular magnetic anisotropy (PMA) [40]. Such modifications of material properties through ionic motion are highly effective in tailoring interfacial characteristics and, consequently, physical and chemical properties [41-46]. Hydrogen-based magneto-ionics is particularly appealing, compared to mostly oxygen-based systems studied so far, due to superior reversibility and speed [40]. Yet, a quantitative understanding of the mechanism is still lacking because of the buried interfaces. Furthermore, besides PMA, other hydrogeninduced magneto-ionic functionalities remain largely unexplored. For example, it would be highly attractive to reversibly control the interfacial DMI, and, in turn, magnetic chirality, via hydrogen transport, especially given the high mobility of hydrogen in solids. Therefore, there is a critical need to confirm and quantify the DMI induced at hydrogen-ferromagnet interfaces and explore its effect on spin textures.
In this article, we report the direct observation of a hydrogen-induced DMI at the surface of ferromagnets at room temperature and a reversible control of the magnetic chirality and spin textures in the ferromagnets. The interfacial DMI induced by chemisorbed hydrogen is quantitatively examined in perpendicularly magnetized $\mathrm{Ni} / \mathrm{Co} / \mathrm{Pd} / \mathrm{W}(110)$ multilayers where the sign and magnitude of the DMI can be tuned by controlling the Pd-layer thickness. When the multilayer has very weak Pd-like DMI (left-handed chirality), we discover a chirality transition of magnetic domain walls in the $\mathrm{Ni} / \mathrm{Co}$ layers from lefthanded to right-handed upon hydrogen chemisorption, indicating that the chemisorbed hydrogen on top of the surfaces introduces finite DMI that favors right-handed chirality, which is supported by density functional theory (DFT) calculations. We further demonstrate that the magnetic chirality of domain walls can be sensitively and reversibly switched between right-handed and left-handed during the chemisorption and desorption of hydrogen. Our results extend the picture of the interfacial DMI to the lightest element and enrich the hydrogen-related design of chiral spintronics and magneto-ionic devices.

\section{RESULTS AND DISCUSSION}

\section{A. Reversible chemisorption and desorption of hydrogen on $\mathrm{Ni}(111)$ and $\mathrm{Co}(0001)$ surfaces}

The essential hydrogen/metal interface is realized via chemisorption of hydrogen on solid surfaces of $\mathrm{Ni}(111)$ and $\mathrm{Co}(0001)$. In the dissociative adsorption of molecular hydrogen used in this study (see Appendix A), prior experimental work showed that the hydrogen atoms adsorb favorably on the top surface (as opposed to diffusion into subsurface binding sites) due to the presence of a chemisorption energy well $[47,48]$, which is also corroborated in more recent analyses using DFT for the case of Ni [49] and Co [50]. Experimental results show that in the case of $\mathrm{Ni}(111)$, the hydrogen atoms occupy threefold hollow sites with a Ni-H bond length of $(1.84 \pm 0.06) \AA$, corresponding to an overlayer-substrate spacing of $(1.15 \pm 0.10) \AA$ [51], and DFT results find that the binding geometry for hydrogen on close-packed Co is very similar [50].

In our experiments, the hydrogen coverage is monitored by measuring the chemisorption-induced work-function shift $\Delta \varphi$ using low-energy electron microscopy (LEEM) [48]. The LEEM is a powerful tool to measure the work function of material surfaces by fitting LEEM IV curves [Fig. 1(a)] [52] (see Appendix B). We observe a work-function increase of $\Delta \varphi \approx 120 \mathrm{meV}$ on a (111)oriented $\mathrm{Ni}$ film upon 0.9 Langmuir hydrogen exposure (180 seconds at $5 \times 10^{-9}$ torr) at room temperature [Fig. 1(a)] (see Appendix A). This significant workfunction shift is in excellent agreement with prior work [53], where a shift of $\Delta \varphi \approx 139 \mathrm{meV}$ was reported to occur upon hydrogen adsorption on a $\mathrm{Ni}(111)$ surface at $41^{\circ} \mathrm{C}$ 

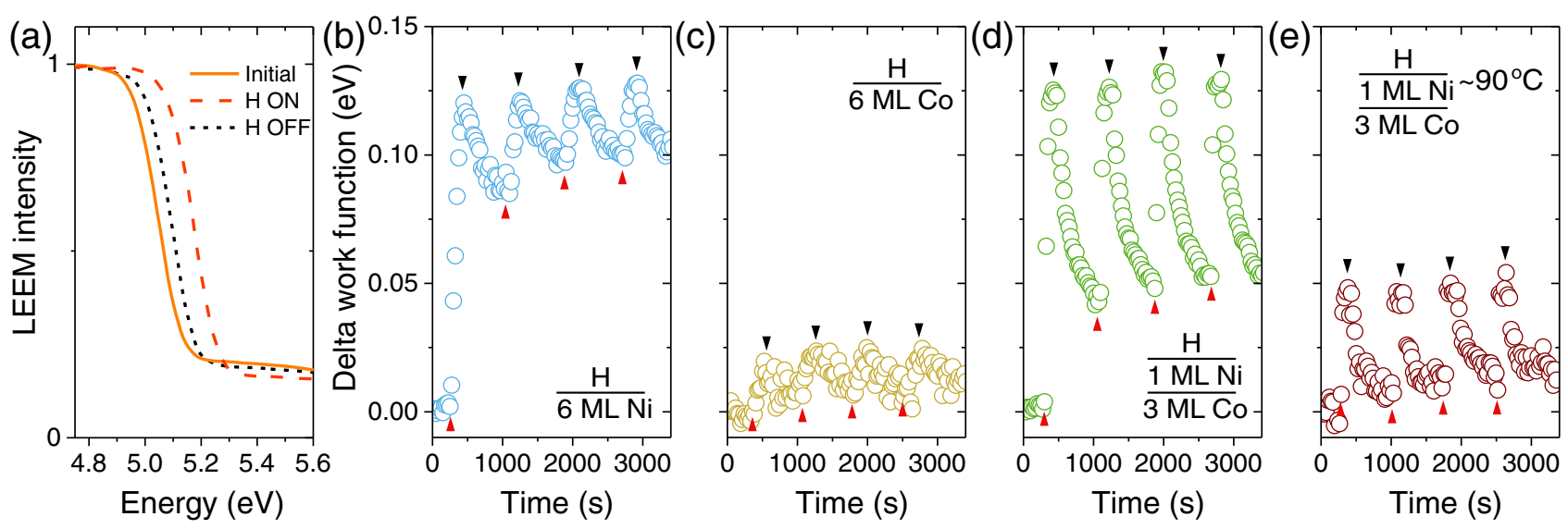

FIG. 1. Room-temperature observation of reversible chemisorption/desorption of atomic hydrogen on metal surfaces. (a) LEEM IV spectra on bare $1 \mathrm{ML} \mathrm{Ni} / 3 \mathrm{ML} \mathrm{Co}$ (initial) and the same surface before and after the hydrogen exposure. Measuring the energy at which the reflectivity drops allows quantification of the work function. (b)-(d) Work-function response on the surface of metals during the presence and absence of hydrogen at room temperature. Red and black triangles indicate the ON and OFF control of the hydrogen leak valve. (b) $6 \mathrm{ML} \mathrm{Ni}$, (c) $6 \mathrm{ML} \mathrm{Co,} \mathrm{(d)} 1 \mathrm{ML} \mathrm{Ni/3} \mathrm{ML} \mathrm{Co.} \mathrm{(e)} \mathrm{Work-function} \mathrm{response} \mathrm{of} 1 \mathrm{ML} \mathrm{Ni} / 3 \mathrm{ML} \mathrm{Co}$ at about $90^{\circ} \mathrm{C}$, indicating about $90 \%$ chemisorption/desorption ratio.

with hydrogen pressure set to $5 \times 10^{-9}$ torr, and with DFT calculations (Appendix D), where a shift of $\Delta \varphi \approx$ $141 \mathrm{meV}$ is found for a full atomic monolayer (ML) $\mathrm{H}$ coverage.

To explore the possible reversibility at room temperature, the evolution of $\Delta \varphi$ is monitored during cycles of turning hydrogen partial pressure $\mathrm{ON}$ and $\mathrm{OFF}$, where the ON state refers to $5 \times 10^{-9}$ torr of hydrogen and OFF refers to the base pressure (below $4 \times 10^{-11}$ torr). For the hydrogencovered $\mathrm{Ni}(111)$ surface, prior flash desorption work identified two desorption maxima around $290-310 \mathrm{~K}\left(\beta_{1}\right.$ state) and $370-380 \mathrm{~K}\left(\beta_{2}\right.$ state) $[53,54]$, where the $\beta_{2}$ feature is only observed at low hydrogen coverage and it saturates at $0.5 \mathrm{ML}$ hydrogen, and the $\beta_{1}$ feature is observed at higher coverage and saturates at 1 ML hydrogen [54]. Here, the depth of the chemisorption well is hydrogen-coverage dependent due to the repulsive planar $\mathrm{H}-\mathrm{H}$ interaction $[48,54]$, which tends to lower the hydrogen binding energy at higher hydrogen coverage [48], resulting in different depths of the chemisorption well as $\Delta E_{\beta_{1}}=0.43 \mathrm{eV}$ and $\Delta E_{\beta_{2}}=0.50 \mathrm{eV}$ [47]. Because the desorption temperature of the $\beta_{1}$ state is just above room temperature, spontaneous hydrogen desorption at room temperature is expected in the ultrahigh vacuum (UHV) condition [53]. Figure 1(b) shows the work-function shift $\Delta \varphi$ on a $\mathrm{Ni}(111)$ surface as a function of time over four $\mathrm{ON}(3 \mathrm{~min}) / \mathrm{OFF}(10 \mathrm{~min})$ cycles. The plot shows the gradual work-function increase of $\Delta \varphi \approx 120 \mathrm{meV}$ during the first hydrogen exposure (0.9 Langmuir) and reversible oscillations of $\Delta \varphi$ during the subsequent ON/OFF cycles with an amplitude of about $\pm 40 \mathrm{meV}$. The known dependence of $\Delta \varphi$ on the hydrogen coverage $[48,53]$ indicates that chemisorption of hydrogen on $\mathrm{Ni}(111)$ is indeed partly reversible at room temperature, and desorption is likely limited to the $\beta_{1}$ state (high hydrogen coverage sites) [53,54]. Consistent with prior literature [53], our result indicates that roughly one-third of the hydrogen can be reversibly chemisorbed and desorbed on a $\mathrm{Ni}(111)$ film surface at room temperature and under ultrahigh vacuum conditions. Note that this coverage ratio may vary as a function of hydrogen dose and pressure [53].

Hydrogen chemisorption also occurs on the $\mathrm{Co}(0001)$ surface, where temperature-programmed thermal desorption measurements indicate desorption maxima with coverage-dependent positions around $325-370 \mathrm{~K}\left(\beta_{1}\right.$ state) and 400-420 K ( $\beta_{2}$ state) [55], somewhat resembling the case of $\mathrm{Ni}(111)$. Similar to $\mathrm{Ni}(111)$, we found that cyclical hydrogen chemisorption and desorption on a Co(111) film is associated with a reversible work-function change, albeit the amplitude is smaller with $\Delta \varphi \approx \pm 20 \mathrm{meV}$. Figure 1(c) plots time-dependent $\Delta \varphi$ measurements over four $\mathrm{ON}$ ( 3 min at $5 \times 10^{-9}$ torr) $/ \mathrm{OFF}(10 \mathrm{~min}$ ) cycles. The observed spontaneous hydrogen desorption from $\mathrm{Co}(0001)$ films at room temperature is consistent with the detailed thermal desorption study of the system reported in Ref. [55].

For the DMI measurements described in detail below, we use $\mathrm{Ni} / \mathrm{Co} / \mathrm{Pd} / \mathrm{W}(110)$ multilayer samples. Here, we first discuss the hydrogen chemisorption properties of such structures. Interestingly, we find that the hydrogen coverage ratio that results in cyclical chemisorption and desorption at room temperature can be greatly enhanced on these multilayer structures, compared to the single-element films described above. Figure 1(d) shows the evolution of $\Delta \varphi$ on the surface of a $\mathrm{Ni}(1) / \mathrm{Co}(3) / \mathrm{Pd}(2) / \mathrm{W}(110)$ multilayer, where the numbers in brackets stand for layer thickness in ML, over an identical hydrogen ON/OFF cycle, as shown in panels (b) and (c). We find that the initial work-function rise of $\Delta \varphi \approx 125 \mathrm{meV}$ upon hydrogen exposure (3 min at $5 \times 10^{-9}$ torr) is comparable to $\Delta \varphi$ observed on $\mathrm{Ni}(111)$ 
( $120 \mathrm{meV})$. However, the amplitude of work-function oscillations during the subsequent hydrogen pressure cycles is around $80 \mathrm{meV}$, about two-thirds of the initial $\Delta \varphi$. This amplitude is almost twice that observed in the thicker (6 ML) Ni(111) film [Fig. 1(b)]. The element Pd is known for its large bulk hydrogen adsorption capacity, and one might surmise that the presence of 2 ML Pd underneath the $\mathrm{Ni} / \mathrm{Co}$ bilayer has something to do with the observed enhancement of the hydrogen-induced work-function change. However, using a $\mathrm{Ni}(1) / \mathrm{Co}(3) / \mathrm{Pd}(20) / \mathrm{W}(110)$ sample with a tenfold thicker Pd layer, we observe that the $\Delta \varphi$ evolution induced by identical hydrogen ON/OFF cycles is almost identical to the sample with just $2 \mathrm{ML}$ Pd (Fig. S1 [56]). This suggests that the large $\Delta \varphi$ ON/OFF ratio originates from the top $\mathrm{Ni} / \mathrm{Co}$ bilayer and not from the Pd layer. Moreover, the enhanced $\Delta \varphi$ ON/OFF ratio at room temperature, compared to the single element films, is likely related to the different hydrogen binding energy on the bilayer composed of a close-packed Co surface with a 1 ML Ni overlayer [57]. Exactly how multilayer structure affects hydrogen desorption at various coverages may merit further investigations using temperature-programmed thermal desorption, which exceeds the scope of this paper. An even greater $\Delta \varphi$ ON/OFF ratio can be achieved on the same $\mathrm{Ni}(1) / \mathrm{Co}(3) / \mathrm{Pd}(2) / \mathrm{W}(110)$ structure at elevated temperatures. Figure 1(e) shows that when the sample is held at $\sim 90^{\circ} \mathrm{C}$, in the hydrogen OFF part of the cycles, the work function nearly fully recovers to the initial value of the hydrogen-free surface. As a result, the ratio of hydrogen coverage extrema in the ON/OFF cycles is on the order of $90 \%$ of the initial work-function rise. This ratio indicates that the sample temperature of $90^{\circ} \mathrm{C}$ is sufficient to activate the hydrogen desorption process related to the second desorption maximum ( $\beta_{2}$ state, the higher-temperature desorption peak in the low hydrogen coverage case), which is comparable to the reported $\beta_{2}$ state at $380 \mathrm{~K}$ in the hydrogen/Ni(111) system [53]. Note that our observed initial work-function rise of $\Delta \varphi \approx 50 \mathrm{meV}$ at $90^{\circ} \mathrm{C}$ is also in reasonable agreement with the value of $\Delta \varphi \approx 40 \mathrm{meV}$ reported in Ref. [53] for $\mathrm{Ni}(111)$ at $89^{\circ} \mathrm{C}$ in $5 \times 10^{-9}$ torr hydrogen.

\section{B. Exploring interfacial DMI induced by chemisorbed hydrogen}

Direct measurement of magnetic chirality is one of the major approaches to unraveling the interfacial DMI [12]. For instance, ground-breaking observations of cycloidal spin spirals using spin-polarized scanning tunneling microscopy have revealed the role of the interfacial DMI on magnetic chirality as well as the period of the spin spirals $[5,6,9,10,13]$. More recently, observation of magnetic chirality in magnetic domain walls also allows the quantification of the magnitude and sign of the interfacial DMI $[16,25,58]$. A particularly versatile approach to measure the DMI at the top interfaces of magnetic multilayers emerges when the magnitude and sign of the effective DMI induced at buried interfaces within the structure can be tuned predictably and accurately. This measurement can be done by using hybrid substrates composed of a bulk crystal coated with a spacer layer where the crystal and spacer induce a DMI of opposite sign, such as $\mathrm{Ir} / \mathrm{Pt}(111)$ [58] or $\mathrm{Pd} / \mathrm{W}(110)$ [27]. The advantage of using a tunable-DMI substrate in this fashion was previously demonstrated in quantifying the DMI induced by chemisorbed oxygen on the $\mathrm{Ni}(111)$ surface [27]. Here, we test the DMI induced by chemisorbed hydrogen on the top surface of $\mathrm{Ni}(1) / \mathrm{Co}(3) / \mathrm{Pd}\left(t_{\mathrm{pd}}\right) / \mathrm{W}(110)$, where the effective DMI in the buried interfaces favors left-handed Néel chirality (Pd-like) at thick Pd thickness $t_{\mathrm{Pd}}$ and righthanded Néel chirality (W-like) [14] at thin $t_{\mathrm{Pd}}$.

What makes this method advantageous for quantifying even rather weak DMI contributions is the fact that the magnitude and sign of the effective DMI of the buried interfaces can be fine-tuned right around the point of null DMI. Here, we track the magnetic chirality evolution upon hydrogen chemisorption on various samples with different initial chirality. We observe a clear hydrogen-induced chirality switching in samples with Pd spacer-layer thickness $t_{\mathrm{Pd}} \sim 2.09 \mathrm{ML}$, where the effective DMI of the hydrogen-free multilayer is weakly Pd-like (left-handed). Figure 2(a) shows a spin-polarized low-energy electron microscopy (SPLEEM) image of the sample in the asgrown state, where the domain-wall magnetization preferentially points from the gray domain $\left(-\mathbf{M}_{\mathbf{z}}\right)$ to the black domain $\left(+\mathbf{M}_{\mathrm{z}}\right)$, corresponding to left-handed Néel chirality. Upon hydrogen chemisorption, Fig. 2(b) shows that the same domain wall evolves to right-handed Néel chirality [now, the domain-wall magnetization predominantly points from the black domain $\left(+\mathbf{M}_{\mathrm{z}}\right)$ to the gray domain $\left(-\mathbf{M}_{\mathrm{z}}\right)$. We denote this switching of the magnetic chirality as the "chirality transition." For a more quantitative analysis, we measure domain-wall chirality in a statistically significant number of image pixels along the domain-wall center line. Defining the parameter $\alpha$ as the angle between the domainwall normal direction $\mathbf{n}$ and the magnetization vector $\mathbf{m}$ at each point along the domain-wall center line [see inset in panel (c)], histograms of the angle $\alpha$ measured from SPLEEM images represent the statistics of domain-wall chirality [25,58]. Figures 2(c) and 2(d) show that, before and after a 0.9 Langmuir hydrogen exposure, the peak at $\alpha \sim 0^{\circ}$ in panel (c) indicates left-handed Néel chirality, whereas the peak at $\alpha \sim 180^{\circ}$ in panel (d) indicates righthanded Néel structure. This statistical approach allows quantification of the chirality transition as shown in Fig. 2(e), where the average domain-wall chirality before and after 0.9 Langmuir hydrogen exposure is plotted for several samples, as a function of Pd spacer-layer thickness $t_{\mathrm{Pd}}$. Note the hydrogen coverage resulting from this dose at room temperature can be roughly estimated as $t_{H}=$ 

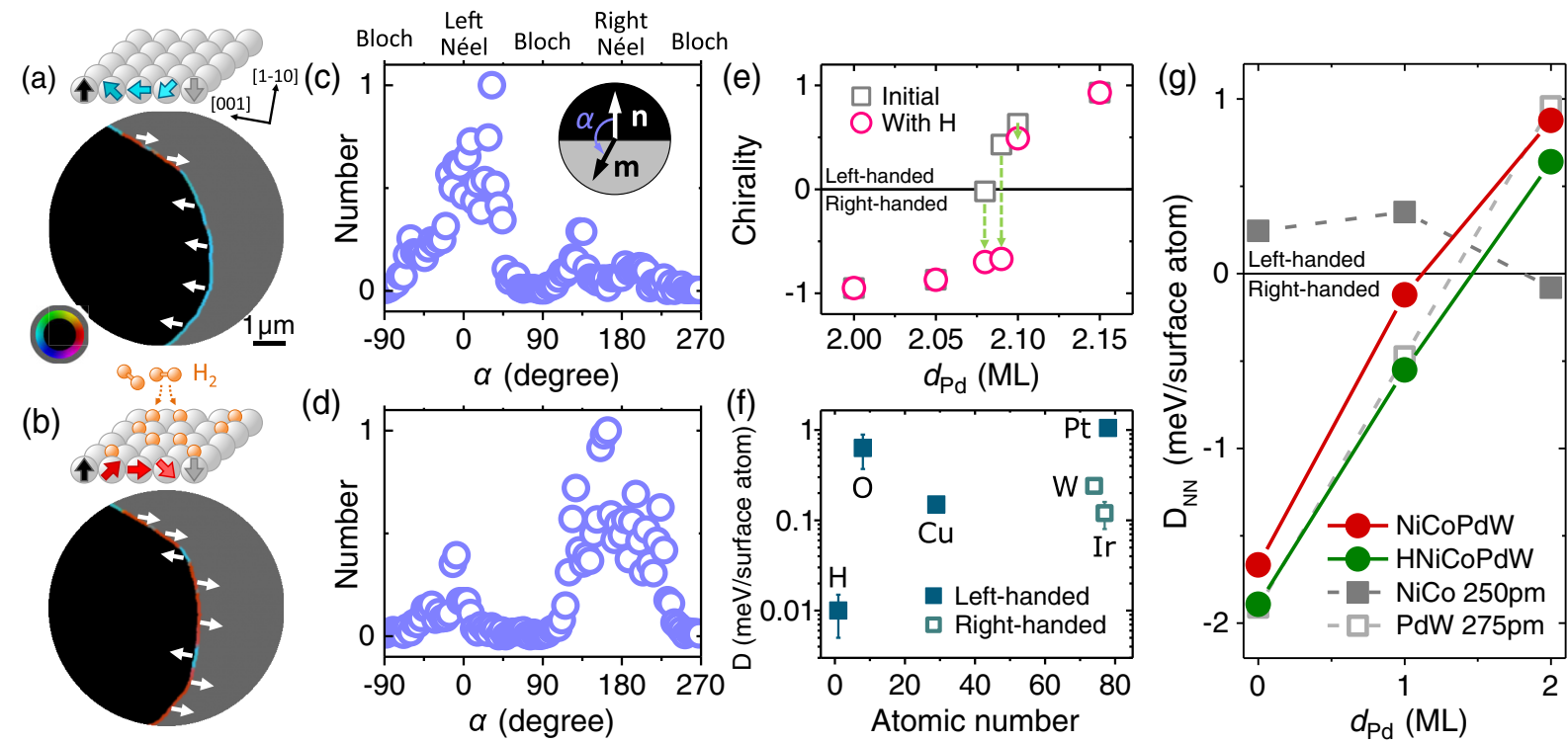

FIG. 2. Exploring chemisorbed-hydrogen-induced Dzyaloshinskii-Moriya interaction. (a,b) Observation of hydrogen-induced domain-wall chirality switching in compound SPLEEM images of 1 ML Ni/3 ML Co/2.09 ML Pd/W(110). (a) As-grown (sketch shows left-handed walls in magnetic layers); (b) with hydrogen exposure at $5 \times 10^{-9}$ torr (sketch shows right-handed walls upon hydrogen chemisorption). Black and gray areas indicate perpendicularly magnetized up and down domains; colors indicate the in-plane orientation of magnetization in the domain-wall region. (c,d) $\alpha$ histogram of the SPLEEM image before (c) and after (d) the hydrogen exposure; $\alpha$ is the angle between domain-wall magnetization $\mathbf{m}$ and the domain-wall normal vector $\mathbf{n}$ (insert). (e) Hydrogen-exposure-dependent evolution of Néel-type chirality at various Pd thicknesses, where the chirality is calculated as $\left(\sum_{-90^{\circ}}^{90^{\circ}}\right.$ counts $-\sum_{90^{\circ}}^{270^{\circ}}$ counts $) /\left(\sum_{-90^{\circ}}^{90^{\circ}}\right.$ counts $+\sum_{90^{\circ}}^{270^{\circ}}$ counts $)$. (f) Summarized values of DMI strength $D$ induced by various elements adjacent to $\mathrm{Ni}$, all measured by the same SPLEEM-based method. (g) DFT calculation of Pd-layer $d_{\mathrm{Pd}}$-dependent DMI strength $D$ from various contributions: Ni/Co layers in the $250 \mathrm{pm}$ structure model (solid squares), Pd/W in the $275 \mathrm{pm}$ model (hollow squares), the sum of both contributions (red dots), and the total contribution including the chemisorbed hydrogen $(\mathrm{H} / \mathrm{Ni} / \mathrm{Co}$ in $250 \mathrm{pm}$ model $+\mathrm{Pd} / \mathrm{W}$ in $275 \mathrm{pm}$ model) (green dots). Lines are guides to the eye.

$(0.6 \pm 0.1)$ ML with respect to the planar atomic density of $\mathrm{Ni}(111)$ (see Appendix A).

When the Pd spacer layer is too thin and the effective DMI remains W-like (right-handed), as in the $t_{\mathrm{Pd}}=2.00 \mathrm{ML}$ and $t_{\mathrm{Pd}}=2.05 \mathrm{ML}$ measurements, then the domain-wall chirality remains completely unaffected by hydrogen chemisorption because the induced DMI at the hydrogen/Ni interface and the effective DMI in $\mathrm{Ni} / \mathrm{Co} /$ thin $-\mathrm{Pd} / \mathrm{W}$ have the same sign (both righthanded). Likewise, when the Pd spacer layer is too thick, as in the $t_{\mathrm{Pd}}=2.15 \mathrm{ML}$ sample, then the Pd-like effective DMI (left-handed) is sufficiently strong to dominate the domain-wall spin texture, and the chirality of the wall remains unaffected even after hydrogen chemisorption because the sign of the effective DMI (left-handed) will not change with the additional weak hydrogen-induced right-handed DMI. However, when the initial DMI is sufficiently weak, as in the samples with $t_{\mathrm{Pd}}=2.08 \mathrm{ML}$, 2.09 ML, and 2.10 ML, then hydrogen chemisorption induces a transition of the domain-wall chirality, clearly revealing the right-handed DMI induced at the hydrogen/ $\mathrm{Ni}(111)$ top interface. Here, the magnitude of the effective DMI is considered to change roughly linearly as a function of submonolayer Pd thickness variation [27] because the typical length scale of the domain walls in our experiment is much larger than the size of third-monolayer $\mathrm{Pd}$ islands on top of the completed second-layer Pd film (see Appendix A). It is interesting to consider whether atomicscale roughness, such as atomic steps surrounding the islands that make up incomplete atomic monolayers, might introduce appreciable DMI. However, if one particular kink site at the edge of a monolayer island induces a certain amount of DMI, then another mirror-symmetric kink site on the opposite side of the island would induce an equal and opposite amount of DMI because of the mirror-symmetric atom arrangement. In the absence of a symmetry-breaking condition, any arrangement of atoms is energetically degenerate with its mirror arrangement. For this reason, populations of left-handed and right-handed DMI-inducing step segments are expected to occur with equal frequencies and must cancel each other. That said, in principle, crystals can be cut in planes that expose chiral kinks such as vicinal surfaces [59-61]. However, the epitaxial layers grown on the $\mathrm{W}(110)$ surface studied here are achiral, and for these symmetry reasons, atomic-scale roughness-induced contributions to the effective DMI would vanish.

Note that the typical Néel-to-Bloch-wall transition near zero DMI is suppressed because a weak in-plane uniaxial 
magnetic anisotropy in this system prevents Blochlike alignment of domain-wall magnetization along the W [1-10] direction [27]. The Néel components of the wall magnetization, however, are clearly sensitive to the sign of the DMI [62]. These results show that chemisorbed hydrogen on top of the Ni(111) surface introduces finite DMI favoring right-handed spin structures, i.e., the same sign of the DMI induced by overlayer Pt, Pd, or oxygen [27]. Note that such stacking order is opposite to the conventional case of ferromagnetic layer (top)/Pt or Pd (bottom) where the left-handedness is favored.

\section{Estimation of the strength of chemisorbed-hydrogen-induced DMI}

The systematic $t_{\mathrm{Pd}}$ spacer-layer thickness-dependent chirality studies summarized in Fig. 2(e) allow us to estimate the magnitude of the hydrogen-induced DMI. The chirality evolution towards right-handedness is observed between 2.08 ML and 2.10 ML during 0.6 ML hydrogen chemisorption. Above 2.10 ML Pd, no significant chirality change can be observed, as the initial, effective, Pd-like DMI now dominates and hydrogeninduced DMI at the $\mathrm{Ni}(111)$ surface can no longer affect the chirality. This approach provides an opportunity to quantify the hydrogen-induced DMI by linking it to the dependence of the initial DMI on the Pd spacer-layer thickness $t_{\mathrm{Pd}}$. Without hydrogen, the achiral state of domain walls, where the effective DMI is essentially zero, occurs at $t_{\mathrm{Pd}} \approx 2.08 \mathrm{ML}$. Upon chemisorption of $0.6 \mathrm{ML}$ hydrogen, the achiral state shifts to $t_{\mathrm{Pd}}=(2.095 \pm 0.004) \mathrm{ML}$. The relative change of the DMI in the $\mathrm{Ni} / \mathrm{Co} / \mathrm{Pd} / \mathrm{W}(110)$ system as a function of the Pd-layer thickness $t_{\mathrm{Pd}}$ was previously quantified as $(0.41 \pm 0.17) \mathrm{meV} /$ surface atom per monolayer $\Delta t_{\mathrm{Pd}}=1 \mathrm{ML}$ [27]. For the DMI values estimated by SPLEEM-image-based evolution of the thickness-dependent domain wall $[27,58]$, we note that the unit of the DMI vectors is given in meV per surface (interface) atom. The measurements summarized in Fig. 2(e) show that the change of effective DMI induced by $0.6 \mathrm{ML}$ hydrogen chemisorption on top of the $\mathrm{Ni} / \mathrm{Co} / \mathrm{Pd} / \mathrm{W}(110)$ multilayer is equivalent to the change of the DMI induced by increasing the Pd spacer-layer thickness by $t_{\mathrm{Pd}}=(0.015 \pm$ 0.004) ML in the absence of hydrogen. Therefore, the DMI induced by the chemisorbed hydrogen on $\mathrm{Ni} / \mathrm{Co} / \mathrm{Pd} / \mathrm{W}$ can be estimated as $(0.41 \pm 0.17) \times(0.015 \pm 0.004 / 0.6 \pm$ $0.1) \mathrm{meV} /$ surface atom $=(0.01 \pm 0.005) \mathrm{meV} /$ surfaceatom for $1 \mathrm{ML}$ equivalent hydrogen coverage. Note that only the chemisorption of hydrogen on the $\mathrm{Ni}$ surface and the role of the hydrogen/Ni interface on the DMI are considered here because the combination of the chemisorption well and the energy barrier of hydrogen bulk diffusion favors the occupation of chemisorption sites [54] in our approach of introducing low-dose (0.9 Langmuir) hydrogen molecules when the sample is held at room temperature.
Figure 2(f) shows a comparison of the DMI induced by various elements adjacent to $\mathrm{Ni}$. For instance, the chemisorbed-hydrogen-induced DMI is much weaker than the chemisorbed-oxygen-induced DMI on $\mathrm{Ni}$, which is $(0.63 \pm 0.26) \mathrm{meV} /$ surface atom at $1 \mathrm{ML}$ equivalent oxygen coverage. The strength of the hydrogen-induced DMI is 1 to 2 orders of magnitude smaller than the DMI induced at $\mathrm{Ni} /$ transition metal interfaces, for example, $\quad D_{\mathrm{Ni} / \mathrm{Cu}+\mathrm{Fe} / \mathrm{Ni}}=(0.15 \pm 0.02) \mathrm{meV} /$ surface atom [16], $\quad D_{\mathrm{Ni} / \mathrm{W}} \approx 0.24 \mathrm{meV} /$ surface atom [62], $\quad D_{\mathrm{Ni} / \mathrm{Ir}}=$ $(0.12 \pm 0.04) \mathrm{meV} /$ surface atom [58], and $D_{\mathrm{Ni} / \mathrm{Pt}}=$ $(1.05 \pm 0.18) \mathrm{meV} /$ surface atom [58]. The hydrogeninduced DMI is also much weaker than the DMI induced at the Co/graphene interface, which is $(0.16 \pm$ $0.05) \mathrm{meV} /$ surface atom [25]. Note that here we only compare DMI measured in SPLEEM-based experiments using methods described in Refs. [16,62,27], to avoid possible systematic measurement biases resulting from the use of different methods [12]. The element-dependent magnitude of the DMI is related to different mechanisms [12]. In the Fert-Levy model $[28,63]$, the DMI scales with the strength of spin-orbit coupling of the adjacent heavy metal [28] and with the degree of orbital hybridization at the $3 d-5 d$ interface as well as the $3 d$ band lineup dictated by Hund's first rule [30]. The graphene-induced DMI is dominated by the Rashba effect, and its magnitude scales with the Rashba coefficient [25]. The oxygen-induced DMI is related to the charge transfer and hybridization at the interface [27,31], and the plausible mechanism of hydrogeninduced DMI is discussed in the next subsection.

Figure 2(g) summarizes the DFT analysis of the $\mathrm{H}$-induced change of the micromagnetic DMI strength $D$ for the system $\mathrm{Ni}(1) / \mathrm{Co}(3) / \mathrm{Pd}\left(d_{\mathrm{Pd}}\right) / \mathrm{W}$ (for details of the calculations, see Appendix D). Since the DMI depends on structural details, the total DMI is calculated as the sum of layer-decomposed contributions. The DMI of $\mathrm{Ni}$ and Co (Pd and W) layers is calculated in the 250 (275) pm lattice constant model. We find that in the absence of Pd, the contribution of $\mathrm{W}$ to the DMI is negative, favoring a right-handed domain wall, consistent with the analysis of Fe on W(110) [14]. The DMI of Pd favors left-handedness (like Pt), and with increasing Pd thickness, $D$ becomes larger and changes sign in $\mathrm{Pd} / W$. The DMI of $\mathrm{Ni}$ and Co favors left-handedness (like Pd), but the magnitude is smaller than that of $\mathrm{Pd}$ and $\mathrm{W}$, consistent with the lower atomic number $\mathrm{Z}$ and the subsequently smaller spin-orbit interaction. Adding up all the contributions, we obtain the total $\mathrm{DMI}$ of $\mathrm{Ni}(1) / \mathrm{Co}(3) / \mathrm{Pd}\left(d_{\mathrm{Pd}}\right) / W$, with a sign change at a Pd thickness of $d_{\mathrm{Pd}} \approx 1 \mathrm{ML}$. In comparison to Fig. $1 \mathrm{H}$ of Ref. [27], the Pd thickness of achirality (thickness of zero DMI) moves by about $0.5 \mathrm{ML}$ due to our refined structural model treating the $\mathrm{Pd}$ and W layers in the $275 \mathrm{pm}$ lattice constant instead of $250 \mathrm{pm}$. Adding $1 \mathrm{ML} \mathrm{H}$ on $\mathrm{Ni}(111)$, we find that the total strength $D$ is reduced, and $\mathrm{H}$ acts like an additional contribution favoring the 

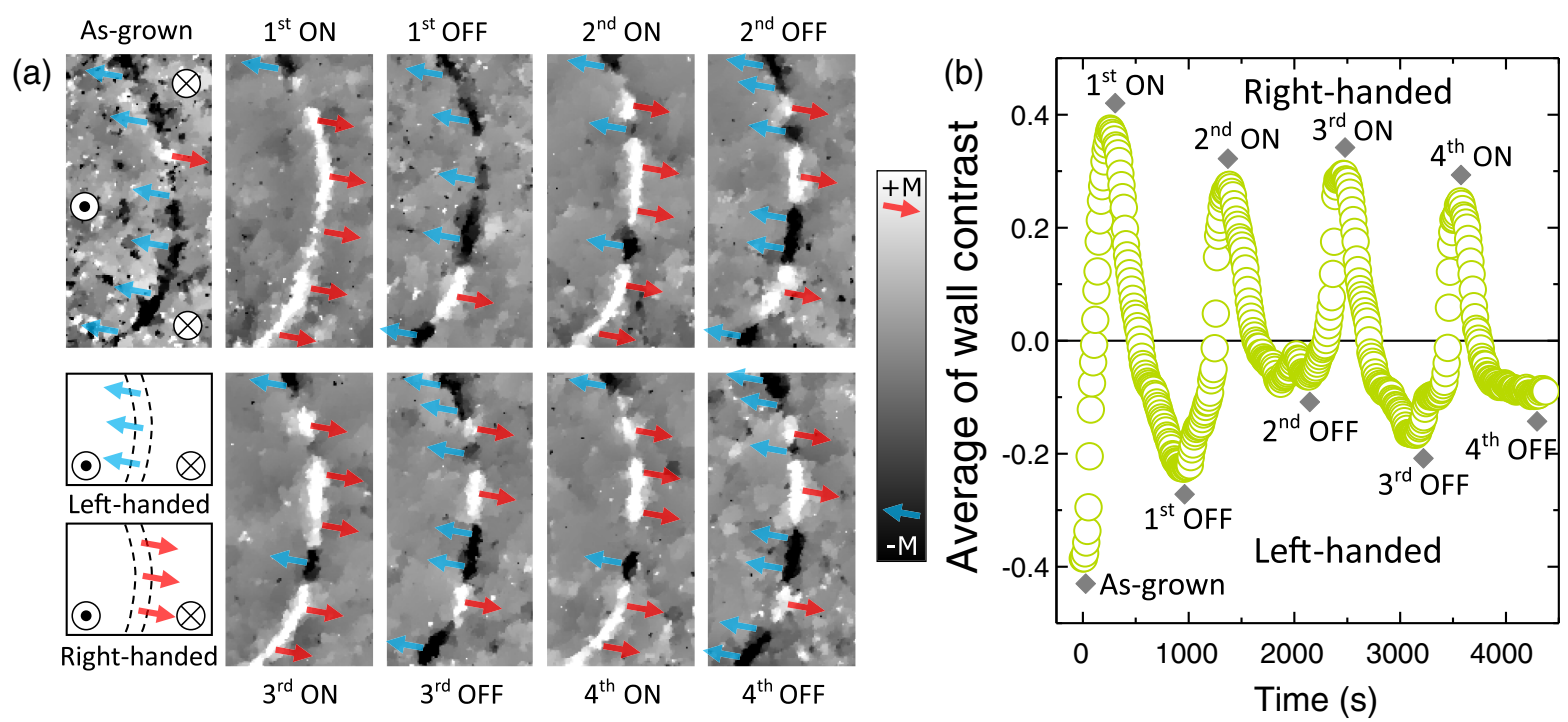

FIG. 3. Reversible switching of magnetic chirality via hydrogen at room temperature. (a) Time sequence of SPLEEM images of a domain wall in a $\mathrm{Ni}(1 \mathrm{ML}) / \mathrm{Co}(3 \mathrm{ML}) / \mathrm{Pd}(2.09 \mathrm{ML}) / \mathrm{W}(110)$ system. Hydrogen status is labeled above and below the images. The inplane magnetization in the domain-wall region is rendered in gray according to the scale bar (right). Domains left and right of the domain wall are perpendicularly magnetized. The magnetization in the left and right regions points up and down, respectively. Magnetic chirality is highlighted by red and cyan arrows (see sketch). The field of view is $2 \mu \mathrm{m} \times 4 \mu \mathrm{m}$. (b) Evolution of magnetic chirality (derived from the average of the wall contrast). Gray diamonds indicate the timing of the images in panel (a).

right-rotating domain wall, thus requiring a larger Pd thickness to reach the achiral point. All of these results are consistent with the experimental facts, but in comparison with the experimental data, the theoretical DMI values are somewhat larger. The experimental thickness to achirality is about $d_{\mathrm{Pd}} \approx 2 \mathrm{ML}$; theoretically, the hydrogen-induced shift equivalent is found to be $0.35 \mathrm{ML}$ of $\mathrm{Pd}$, while in the experiment, it is about $0.015 \mathrm{ML}$ of Pd. We attribute these quantitative discrepancies to the difficulty in accurately modeling the true structure of the materials stack.

\section{Hydrogen-assisted reversible control of the chirality}

The observation of substantial reversibility of hydrogen chemisorption by desorption in clean UHV at room temperature, together with the observed hydrogen-induced switching of domain-wall chirality, suggests the possibility to reversibly switch the domain-wall chirality by hydrogen chemisorption and desorption cycles. To test this possibility, we use SPLEEM to continuously monitor the domain-wall magnetization in a $\mathrm{Ni}(1 \mathrm{ML}) / \mathrm{Co}(3 \mathrm{ML}) / \mathrm{Pd}(2.09 \mathrm{ML}) /$ $\mathrm{W}(110)$ multilayer, while periodically cycling between $5 \times 10^{-9}$ torr hydrogen pressure for 3 minutes and negligible hydrogen pressure (UHV base pressure) for 10 minutes. Figure 3(a) shows the evolution of the domain-wall chirality in the four cycles, where the chirality switches from predominantly left-handed to predominantly right-handed upon the first hydrogen chemisorption [see the definition of chirality in Fig. 3(a)], and the chirality partially evolves toward left-handedness/right-handedness during
"H OFF"/"H ON" states for the rest of the cycles. Figure 3(b) shows the statistics of this domain-wall switching experiment, tracking reversibility of the chirality over four cycles at room temperature (see Appendix C). Note that here the chirality is illustrated by the average of the normalized SPLEEM contrast pixel by pixel along the domain wall region, which is different from how the chirality is calculated in Fig. 2(e). This magnetic chirality measurement is correlated with a hydrogen coverage measurement, as monitored by tracking the work-function change of $+120 \mathrm{meV}$ for the first $\mathrm{H}-\mathrm{ON}$ state and $\pm 80 \mathrm{meV}$ for the subsequent cycles. These results indicate that the hydrogen coverage changes shown previously in Fig. 1(d) indeed reversibly affect the DMI of the system so as to switch the sign of the effective DMI as well as the domain-wall chirality. In this experiment, the chirality reversal during the H-OFF state is imperfect in the sense that a small fraction of domain-wall sections remain in the right-handed state corresponding to the hydrogen-induced DMI. It is plausible that these minor imperfections in the chirality switching are due to a combination of defect-induced pinning and the weaker DMI associated with residual hydrogen coverage due to incomplete desorption in the 10-minute OFF cycles.

In the end, the physical origin of the chemisorbedhydrogen-induced change in the DMI is the change in the spatial asymmetry of the wave function in the transition metals, especially in $\mathrm{Ni}$ surface atoms. The hydrogen causes this change by a slight outward relaxation of the nickel position above the Co film (see Appendix D), 
induced by a small charge transfer from nickel to hydrogen and by the polarization and rehybridization of the orbitals in nickel. The electrical surface dipole moment and the work function are the corresponding physical quantities that provide information about these changes. In fact, a linear relationship between the work-function change and the DMI was demonstrated for $\mathrm{Pt} / \mathrm{Co} /$ transitionmetal trilayers [64], and a linear relationship between the electronegativity, the electric dipole moment, and the DMI was proposed in Ref. [29]. To shed more light on these relationships, we compare the changes in DMI $(\mathrm{H}: 0.01 \pm 0.005 \mathrm{meV} / 1 \mathrm{ML} \mathrm{H}, \quad \mathrm{O}: 0.63 \pm 0.26 \mathrm{meV} /$ $1 \mathrm{ML}$ O), work-function (H: $\sim 0.12 \mathrm{eV}, \mathrm{O}: \sim 0.70 \mathrm{eV}$ ), and Pauling electronegativity differences $(\mathrm{H}: 0.29$, O: 1.53 , relative to $\mathrm{Ni}$ ) between the hydrogen $(\mathrm{H}:)$ and oxygen $(\mathrm{O}:)$ [27] terminated NiCoPdW systems (the electronegativities of $\mathrm{H}, \mathrm{O}$, and $\mathrm{Ni}$ are 2.20, 3.44, and 1.91, respectively). Note that the electronegativity of both hydrogen and oxygen is larger than that of nickel, which is consistent with the measurements where the work function on the surface of $\mathrm{NiCoPdW}$ increases upon adsorption of both hydrogen and oxygen. Also, the electronegativity of oxygen is larger than that of hydrogen, and the strength of the right-handed DMI increases, more significantly for the oxygen system than for the hydrogen system. Indeed, the ratios of the workfunction shift $\left(R_{\Delta \varphi}=0.70 / 0.12 \approx 5.8\right)$ and the changes of the electronegativities $\left(R_{\Delta \chi}=1.53 / 0.29 \approx 5.3\right)$ between the oxygen system and the hydrogen system are about the same, i.e., $R_{\Delta \varphi} / R_{\Delta \chi} \approx 1$. Evidently, for these systems, the change of the electronegativity is an excellent measure for the estimation of the change of the work function. Considering the ratios between the change in DMI to the change in work function, one obtains $0.63 \mathrm{meV} / 0.70 \mathrm{eV}$ for the oxygen system, i.e., a change in DMI of about $1 \mathrm{meV}$ per $1 \mathrm{eV}$ change in work function. For the hydrogen system, one gets a value that is more than 10 times smaller $(0.01 \mathrm{meV} / 0.12 \mathrm{eV})$. Also, the same ratios are obtained if the work-function changes are replaced by the electronegativities. Testing whether one-to-one relationships between the DMI and work-function change [64] or electronegativity [29] hold more generally goes beyond the scope of this paper. We speculate that either the changes induced by the oxygen are so strong that we are no longer in the linear regime or the DMI value of the hydrogen system is so small due to the compensation of the different contributions.

Chemisorption of hydrogen occurs on many transition metals; in particular, a considerable hydrogeninduced dipolar moment appears (via the observation of a work-function shift) on the surfaces of ferromagnetic metals such as cobalt, nickel, and iron, or $4 d / 5 d$ metals, and we expect that chemisorbed-hydrogeninduced DMI can generally be observed on ferromagnetic thin films. However, the reversibility demonstrated in Figs. 1 and 3 may require additional testing for each specific case. Furthermore, we note that hydrogen is the lightest element of the periodic table. It is tiny, has no chemical aggressiveness, and is not as electronegative as oxygen. In this sense, hydrogen is rather gentle, and it might be the ideal chemical element that enables the best control of the DMI needed to change the spatial properties of spin textures such as skyrmions, much more precisely than oxygen.

The sensitive and reversible switching of the DMI and chiral spin texture via hydrogen chemisorption is highly relevant for chiral spintronics, such as racetrack memories based on domain walls or skyrmions, where hydrogeninduced DMI may be used to manipulate the chiral domainwall motion by controlling the chirality, or to sensitively control the skyrmion size over orders of magnitude [22], a feature particularly interesting for neuromorphic computing. One key advantage is that the switching via chemisorption may be done in a tunable and contactless fashion, without requiring electrical leads to be attached to the device. This is particularly attractive for complex device geometries such as three-dimensional racetrack memory [65] and networks [66] involving numerous domain walls or skyrmions. Note that the role of hydrogen on the DMI is not limited to surfaces, as demonstrated in this paper, and rich possibilities exist where hydrogen may occupy sites inside the bulk, such as hydrogenated $\mathrm{Fe} / \operatorname{Ir}(111)$, which may also alter the DMI via lattice spacing changes [36]. These hydrogen-based results are also relevant to the emerging field of magneto-ionics, which has so far been largely based on oxygen ions and vacancies. They not only significantly expand on the magnetic functionalities that can be controlled magneto-ionically but also offer exciting potentials for completely reversible and energy-efficient switching. We speculate that the hydrogenation of interfaces of films consisting of magnetic and high-spin-orbit materials opens a vista to new magneto-ionic or memristive functionalities. For example, electrical control of hydrogen coverage through magneto-ionic means could lead to a change of DMI and, consequently, spin textures, such as chiral domain-wall motion or skyrmion size; in the former case, the chiral domain-wall motion may be useful for magnetic logic devices as well as artificial synapses [67], and in the latter case, magnetoresistive properties of skyrmion-based devices may be relevant for memristors. Importantly, using the chemisorption mechanism, the hydrogen ions can be driven across atomic distances to contact the relevant ferromagnet surface in solid-state devices (and reduce to atomic form [40]) but without actually penetrating the surface and causing any irreversible changes, thus leading to excellent reversibility, endurance, and potential for high speed.

\section{CONCLUSION}

In summary, we report a direct and quantitative observation of a hydrogen chemisorption-induced DMI on 
ferromagnet surfaces at room temperature, which can be used to sensitively and reversibly switch chiral domain walls. We find that the chemisorption/desorption ratio of hydrogen is greatly enhanced by combining $1 \mathrm{ML} \mathrm{Ni}$ and $3 \mathrm{ML}$ Co at the top of the multilayers, and even under constant room-temperature conditions, the reversibility of hydrogen chemisorption can reach as much as two-thirds of the initial hydrogen coverage. We observe that the hydrogen chemisorption induces a finely controllable, reversible, and nonvolatile chirality transition of magnetic domain walls in the $\mathrm{Ni} / \mathrm{Co} / \mathrm{Pd} / \mathrm{W}(110)$ system at room temperature. This chirality control is attributed to the hydrogeninduced DMI, which is experimentally quantified. These results help to advance the understanding of the DMI induced by light elements and open up new device potentials in chiral spintronics and magneto-ionics.

\section{ACKNOWLEDGMENTS}

We thank Hongying Jia and Tianping Ma for insightful discussions, and Steven E. Zeltmann for help with the TVD implementation. This work has been supported in part by the NSF (DMR-1905468 and DMR-2005108), the University of California Office of the President Multicampus Research Programs and Initiatives (MRP-17-454963), and Spintronic Materials for Advanced InfoRmation Technologies (SMART) (2018-NE-2861), one of seven centers of nCORE, a Semiconductor Research Corporation program, sponsored by the National Institute of Standards and Technology (NIST). Work at the Molecular Foundry was supported by the Office of Science, Office of Basic Energy Sciences, of the U.S. Department of Energy under Contract No. DE-AC02-05CH11231. Work at Nanjing University has been supported by the National Key R\&D Program of China (Grant No. 2017YFA0303202), and the National Natural Science Foundation of China (Grants No. 11734006 and No. 11974165). R. L. C., A. K. S., and R. W. acknowledge financial support by the European Union via an International Marie Skłodowska-Curie Fellowship (Grant No. 748006-SKDWONTRACK). C. O. acknowledges support from the U.S. Department of Energy Early Career Research Program. S. B. acknowledges financial support from the DARPA TEE program through Grant MIPR\#HR0011831554 from DOI, from Deutsche Forschungsgemeinschaft (DFG) through SPP 2137 "Skyrmionics" (Project BL 444/16) and the Collaborative Research Centers SFB 1238 (Project C01). A. L. F. C. and A. K. S. thank the Advanced Research Projects AgencyEnergy (ARPA-E), U.S. Department of Energy, under Award No. DE-AR0000664.

\section{APPENDIX A: SAMPLE PREPARATION AND HYDROGEN EXPOSURE}

The SPLEEM experiments were performed at the National Center for Electron Microscopy of the
Lawrence Berkeley National Laboratory. All samples were grown in the SPLEEM chamber under ultrahighvacuum (UHV) conditions, with a base pressure better than $4.0 \times 10^{-11}$ torr. The $\mathrm{W}(110)$ substrate was cleaned by cycles of flashing to $1950^{\circ} \mathrm{C}$ in $3.0 \times 10^{-8}$ torr $\mathrm{O}_{2}$, followed by a final flashing at the same temperature to remove oxygen. $\mathrm{Ni}, \mathrm{Co}$, and $\mathrm{Pd}$ layers were deposited by physical vapor deposition from electron-beam evaporators when the substrate is held at room temperature, and the film thicknesses of Ni, Co, and Pd layers were calibrated by monitoring the oscillations of the LEEM image intensity associated with atomic layer-by-layer growth [27]. In contrast to the significantly larger $\mathrm{Pd}$ island structures grown at high temperature [68], in our roomtemperature sample preparations, the strong layer-bylayer electron reflectivity oscillations, together with the observation of featureless LEEM images, indicate that the typical size of next-monolayer Pd islands at the growth front of the Pd films is smaller than the spatial resolution at the magnification used in these experiments (image pixel size is about $22 \mathrm{~nm}$ at the $10 \mu \mathrm{m}$ field of view used in this work). Note that the Pd thickness with zero effective DMI $\left(t_{\mathrm{Pd}} \approx 2.08 \mathrm{ML}\right)$ here is slightly thinner than that reported in Ref. [27] ( $t_{\mathrm{Pd}} \approx 2.46 \mathrm{ML}$ ), which is possibly due to slight differences in experimental conditions.

Hydrogen exposures were realized by leaking of highpurity hydrogen $(99.999 \%)$ at a pressure of $5 \times 10^{-9}$ torr. The pressure reading of hydrogen on the ionization gauge has been corrected by a factor of 0.46 . No noticeable change was observed in the LEED pattern upon hydrogen chemisorption at room temperature, which is consistent with Ref. [51]. On Ni(111), the maximum work-function shift occurs at the hydrogen coverage of 0.5-0.6 ML [51], and volumetric measurements reveal that the saturation coverage of chemisorbed hydrogen on $\mathrm{Ni}(111)$ is about $0.7 \mathrm{ML}$ at room temperature [53]. Therefore, the hydrogen coverage on the surface of $\mathrm{Ni} / \mathrm{Co} / \mathrm{Pd} / \mathrm{W}(110)$ is estimated based on the work-function shift measurement with a maximum work-function shift $[\Delta \varphi \approx 125 \mathrm{meV}$, Fig. 1(d)], which roughly corresponds to a $0.5-0.7 \mathrm{ML}$ hydrogen overlayer.

\section{APPENDIX B: TIME-DEPENDENT WORK-FUNCTION MEASUREMENT}

The work function is determined by fitting the LEEM IV spectrum [image intensity vs incident energy of electrons; see Fig. 1(a)] with a complementary error function erfc (start voltage) [52]. The value where the dropoff occurs, $V_{S}^{0}$, represents a measurement of the sample work function given by $\phi_{\text {sample }}=V_{S}^{0}+E_{C}^{0}$, where $E_{C}^{0}$ represents the peak of the electron distribution emitted from our photocathode (p-type $\mathrm{GaAs}$ crystal activated with $\mathrm{CsO}_{x}$ ). The emission of the GaAs cathode of SPLEEM is set to $100 \mathrm{nA}$ to optimize the energy spread to about $180 \mathrm{meV}$ (full width at half 
maximum) and $E_{C}^{0} \sim 1.4-1.5 \mathrm{eV}$, measuring a reference surface such as highly oriented pyrolytic graphite. Timedependent work-function measurements were performed by recording the reflectivity of low-energy electrons while sweeping the start voltage in a loop (Fig. S2 [56]). In order to record the work-function changes during hydrogen adsorption and desorption at the surface, the start voltage was swept from $1.5 \mathrm{~V}$ below to about $2 \mathrm{~V}$ above the intensity dropoff using $50-\mathrm{mV}$ voltage steps and an image integration time of $250 \mathrm{~ms}$. Relative changes in the work function over time can be detected with very-high sensitivity down to about $5 \mathrm{mV}$ given by the shift of the centroid of the Gaussian distribution extracted by the erfc (start voltage) fitting.

\section{APPENDIX C: TIME-DEPENDENT IN-PLANE DOMAIN-WALL ANALYSIS}

Because of the noise present in the individual in-plane domain-wall images, we used standard image denoising methods to provide a more accurate estimate for the magnetization presented in Fig. 3. The measured images were denoised by $3 \mathrm{D}$ total variational denoising (3D-TVD), using a MATLAB implementation and $3 \mathrm{D}$ extension to the methods given in Ref. [69]. After normalizing the data to have a mean intensity of zero and a standard deviation of 1 , we used regularization parameters of $\mu=[2,2,1]$ and $\lambda=[1 / 8,1 / 8,1 / 16]$ for the dimensions of $x, y$ and time, respectively. FISTA acceleration was used to speed up convergence. The regularization was applied isotropically to the $x$ and $y$ directions. After the TVD was applied, we normalized the images to have a mean of zero and the boundary contrast to have an approximate range of -1 to +1 .

\section{APPENDIX D: DFT RESULTS AND ANALYSIS}

All first-principles calculations of clean and hydrogencovered $\mathrm{Ni}(1) / \mathrm{Co}(3) / \mathrm{Pd}(\mathrm{n}) / \mathrm{W}(110)$ systems were based on DFT. All calculations, approximations to exchange and correlation functionals, first-principles methods, computational procedures, and computational parameters are consistent with the calculations in Ref. [27]. We have investigated systems by varying the thickness of $\mathrm{Ni}$ between zero and three atomic layers and of Pd between zero and three layers, keeping the number of Co layers fixed at three atomic layers.

Structural model. Since the substrate is a (110) oriented bcc W crystal, Pd, Co and Ni grow as fcc (111), and the lattice constants of $\mathrm{Ni}$ and $\mathrm{Co}$ are considerably different from $\mathrm{Pd}$, we expect that some adaptation of the atoms and some strain release may take place on the way from the $\mathrm{W}$ surface to the Ni layer, which is difficult to capture in full detail in our structural model. We have modeled all systems by pseudomorphically (111) stacked transition-metal layers with a stacking order of the preferred bulk ordering for each metal. The system was approximated by an asymmetric stack of layers, where the $\mathrm{W}$ substrate is modeled by five layers of $\mathrm{W}$. We studied results for two different structural models: (a) with the in-plane lattice constants $a_{\mathrm{IP}}=$ $250 \mathrm{pm}$ and (b) with $a_{\mathrm{IP}}=275 \mathrm{pm}$; the former is related to the average of the experimental bulk lattice constant of $\mathrm{Ni}\left(a_{\mathrm{Ni}}=352 \mathrm{pm}\right.$ and $\left.a_{\mathrm{IPNi}}=a_{\mathrm{Ni}} / \sqrt{2}=249 \mathrm{pm}\right)$ and hcp-Co $\left(a_{\mathrm{IPCo}}=251 \mathrm{pm}\right)$, and the latter to the average of the experimental bulk lattice constant of fcc-Pd $\left(a_{\mathrm{Pd}}=389 \mathrm{pm}\right.$ and $\left.a_{\mathrm{IPPd}}=275 \mathrm{pm}\right)$ and the cubic bulk lattice constant of W $\left(a_{W}=316 \mathrm{pm}\right.$ and $a_{\mathrm{IPW}}=a_{\mathrm{W}} \sqrt{3} / 2=274 \mathrm{pm}$ ). To most accurately model the $\mathrm{W}(110)$ substrate, we adjusted the $\mathrm{W}$ interlayer distances to obtain the correct experimental bulk volume. For simplicity, we considered a $100 \%$ coverage of $\mathrm{H}$ on the $\mathrm{Ni}$ surface. In the following, we relate all surface-related quantities to the results of the $250 \mathrm{pm}$ model and all $\mathrm{W}$ and Pd related results to the $275 \mathrm{pm}$ model.

Results. On the basis of structural optimization by energy minimization, we found the following: (i) $\mathrm{H}$ adsorbs on the fcc hollow sites for the $a=250 \mathrm{pm}$ model. (ii) $\mathrm{H}$ reduces the bond strength between $\mathrm{Ni}$ and $\mathrm{Co}$ and increases the interlayer distance from $198 \mathrm{pm}$ to $207 \mathrm{pm}$. This result is basically independent of the number of Pd layers. (iii) $\mathrm{H}$ substantially reduces the magnetic moment of $\mathrm{Ni}$ from $0.72 \mu_{B}$ to $0.21 \mu_{B}$ and, by a tiny amount $(2.2 \%)$, the magnetic moment of the first $\mathrm{Co}$ layer adjacent to $\mathrm{Ni}$. (iv) The work function of $\mathrm{W}$ in the $275 \mathrm{pm}$ model amounts to $\varphi_{W}=5.40 \mathrm{eV}$, in good agreement with the experimental work function of $\mathrm{W}(110), \varphi_{W}=5.22 \mathrm{eV}$. (v) The work function of $\mathrm{Ni} / \mathrm{Co} / \mathrm{Pd} / \mathrm{W}$ in the $250 \mathrm{pm}$ model amounts to $\varphi_{\mathrm{Ni}}=5.28 \mathrm{eV}$, in good agreement with the experimental work function of $\mathrm{Ni}(111), \varphi_{\mathrm{Ni}}=5.35 \mathrm{eV}$. The result is practically independent of the number of Pd layers. (vi) The work-function change $\Delta \varphi$, upon adsorption of $\mathrm{H}$ on the surface of $\mathrm{Ni}$ for the system $\mathrm{Ni}(1) / \mathrm{Co}(3) / \mathrm{Pd}(2) / \mathrm{W}$, is $\Delta \varphi \approx 141 \mathrm{meV}$. It is in excellent agreement with the experimentally measured value $\Delta \varphi \approx 139 \mathrm{meV}$ for $\mathrm{H}$ on $\mathrm{Ni}(111)$ at $41^{\circ} \mathrm{C}$ and $5 \times 10^{-9}$ torr hydrogen pressure [53] and, within this work for which $\Delta \varphi \approx 125 \mathrm{meV}$, was found, except for a smaller coverage of about 0.6 ML.

$D M I$. In determining the interface DMI and its changes upon hydrogen coverage, we proceeded analogously to the experimental procedure. We first calculated, from DFT, the DMI strength $D$ [61] of the micromagnetic DMI tensor, which was then expressed in the language of the atomistic spin model, as in the experiment, with the energy expression $E_{\mathrm{DM}}=\sum_{\langle i j\rangle} \mathbf{D}_{i j} \cdot\left(\mathbf{S}_{i} \times \mathbf{S}_{j}\right)$ in terms of an effective DMI vector $\mathbf{D}_{i j}$ between nearest-neighbor (NN) pairs. Here, $\langle i j\rangle$ denotes the summation over unique pairs of nearest-neighbor atoms. The effective atomistic nearest-neighbor strength $D_{\mathrm{NN}}$ and the micromagnetic DMI strength $D$ are related as $D_{\mathrm{NN}}=1 /\left(3 \pi a_{\mathrm{IP}}\right) D$, with $a_{\mathrm{IP}}=251 \mathrm{pm}$ (see the in-plane lattice constant $a_{\|}$used to determine the dipolar energy [27]). Summing up all pairs $\langle i j\rangle$ across the domain wall, 
$E_{\mathrm{DM}}$ corresponds to the DMI energy in one domain wall and relates to the size of the effective nearest-neighbor DMI vector $D_{\mathrm{NN}}$ as $E_{\mathrm{DM}}=-\sqrt{3} \pi\left|D_{\mathrm{NN}}\right|$.

[1] I. Dzyaloshinsky, A Thermodynamic Theory of Weak Ferromagnetism of Antiferromagnetics, J. Phys. Chem. Solids 4, 241 (1958).

[2] T. Moriya, Anisotropic Superexchange Interaction and Weak Ferromagnetism, Phys. Rev. 120, 91 (1960).

[3] M. Uchida, Y. Onose, Y. Matsui, and Y. Tokura, Real-Space Observation of Helical Spin Order, Science 311, 359 (2006).

[4] X. Z. Yu, Y. Onose, N. Kanazawa, J. H. Park, J. H. Han, Y. Matsui, N. Nagaosa, and Y. Tokura, Real-Space Observation of a Two-Dimensional Skyrmion Crystal, Nature (London) 465, 901 (2010).

[5] M. Bode, M. Heide, K. von Bergmann, P. Ferriani, S. Heinze, G. Bihlmayer, A. Kubetzka, O. Pietzsch, S. Blugel, and R. Wiesendanger, Chiral Magnetic Order at Surfaces Driven by Inversion Asymmetry, Nature (London) 447, 190 (2007).

[6] S. Heinze, K. von Bergmann, M. Menzel, J. Brede, A. Kubetzka, R. Wiesendanger, G. Bihlmayer, and S. Blügel, Spontaneous Atomic-Scale Magnetic Skyrmion Lattice in Two Dimensions, Nat. Phys. 7, 713 (2011).

[7] N. Nagaosa and Y. Tokura, Topological Properties and Dynamics of Magnetic Skyrmions, Nat. Nanotechnol. 8, 899 (2013).

[8] F. S. Zheng, F. N. Rybakov, A. B. Borisov, D. S. Song, S. S. Wang, Z. A. Li, H. F. Du, N. S. Kiselev, J. Caron, A. Kovacs, M. L. Tian, Y.H. Zhang, S. Blügel, and R.E. Dunin-Borkowski, Experimental Observation of Chiral Magnetic Bobbers in B20-Type FeGe, Nat. Nanotechnol. 13, 451 (2018).

[9] S. Meckler, N. Mikuszeit, A. Pressler, E. Y. Vedmedenko, O. Pietzsch, and R. Wiesendanger, Real-Space Observation of a Right-Rotating Inhomogeneous Cycloidal Spin Spiral by Spin-Polarized Scanning Tunneling Microscopy in a Triple Axes Vector Magnet, Phys. Rev. Lett. 103, 157201 (2009).

[10] R. Wiesendanger, Nanoscale Magnetic Skyrmions in Metallic Films and Multilayers: A New Twist for Spintronics, Nat. Rev. Mater. 1, 16044 (2016).

[11] A. Fert, N. Reyren, and V. Cros, Magnetic Skyrmions: Advances in Physics and Potential Applications, Nat. Rev. Mater. 2, 17031 (2017).

[12] W. Jiang, G. Chen, K. Liu, J. Zang, S. G. E. te Velthuis, and A. Hoffmann, Skyrmions in Magnetic Multilayers, Phys. Rep. 704, 1 (2017).

[13] E. Y. Vedmedenko, L. Udvardi, P. Weinberger, and R. Wiesendanger, Chiral Magnetic Ordering in TwoDimensional Ferromagnets with Competing DzyaloshinskyMoriya Interactions, Phys. Rev. B 75, 104431 (2007).

[14] M. Heide, G. Bihlmayer, and S. Blügel, DzyaloshinskiiMoriya Interaction Accounting for the Orientation of Magnetic Domains in Ultrathin Films: Fe/W(110), Phys. Rev. B 78, 140403(R) (2008).
[15] A. Thiaville, S. Rohart, É. Jué, V. Cros, and A. Fert, Dynamics of Dzyaloshinskii Domain Walls in Ultrathin Magnetic Films, Europhys. Lett. 100, 57002 (2012).

[16] G. Chen, J. Zhu, A. Quesada, J. Li, A. T. N'Diaye, Y. Huo, T. P. Ma, Y. Chen, H. Y. Kwon, C. Won, Z. Q. Qiu, A. K. Schmid, and Y.Z. Wu, Novel Chiral Magnetic Domain Wall Structure in $\mathrm{Fe} / \mathrm{Ni} / \mathrm{Cu}(001)$ Films, Phys. Rev. Lett. 110, 177204 (2013).

[17] H.-B. Braun, Topological Effects in Nanomagnetism: From Superparamagnetism to Chiral Quantum Solitons, Adv. Phys. 61, 1 (2012).

[18] K.-S. Ryu, L. Thomas, S.-H. Yang, and S. Parkin, Chiral Spin Torque at Magnetic Domain Walls, Nat. Nanotechnol. 8, 527 (2013).

[19] S. Emori, U. Bauer, S.-M. Ahn, E. Martinez, and G. S. D. Beach, Current-Driven Dynamics of Chiral Ferromagnetic Domain Walls, Nat. Mater. 12, 611 (2013).

[20] R. Blasing, T. P. Ma, S. H. Yang, C. Garg, F. K. Dejene, A. T. N'Diaye, G. Chen, K. Liu, and S. S. P. Parkin, Exchange Coupling Torque in Ferrimagnetic Co/Gd Bilayer Maximized near Angular Momentum Compensation Temperature, Nat. Commun. 9, 4984 (2018).

[21] K. M. Song, J.-S. Jeong, B. Pan, X. Zhang, J. Xia, S. Cha, T.-E. Park, K. Kim, S. Finizio, J. Raabe, J. Chang, Y. Zhou, W. Zhao, W. Kang, H. Ju, and S. Woo, Skyrmion-Based Artificial Synapses for Neuromorphic Computing, Nat. Electron. 3, 148 (2020).

[22] In the limit of stable skrymions, the skyrmion radius $R$ is inversely proportional to the DMI strength $D$, $R \propto 1 / \sqrt{\left(\gamma^{2}-D^{2}\right)}$, where $\gamma=4 \sqrt{A K} / \pi$, with $A$ the exchange stiffness and $K$ the effective magnetic anisotropy; see S. Komineas, C. Melcher, and S. Venakides, Chiral Skyrmions of Large Radius, Physica (Amsterdam) 418D, 132842 (2021). Thus, with a fine-tuning of $D$ close to $\gamma$, the radius of the skyrmion may be tailored by orders of magnitude.

[23] D. S. Chaves, F. Ajejas, V. Krizakova, J. Vogel, and S. Pizzini, Oxidation Dependence of the DzyaloshinskiiMoriya Interaction in $\mathrm{Pt} / \mathrm{Co} / \mathrm{MO}_{x}$ Trilayers $(M=\mathrm{Al}$ or Gd), Phys. Rev. B 99, 144404 (2019).

[24] H. T. Nembach, E. Jue, E. R. Evarts, and J. M. Shaw, Correlation between Dzyaloshinskii-Moriya Interaction and Orbital Angular Momentum at an Oxide-Ferromagnet Interface, Phys. Rev. B 101, 020409(R) (2020).

[25] H. Yang, G. Chen, A. A. C. Cotta, A. T. N'Diaye, S. A. Nikolaev, E. A. Soares, W. A. A. Macedo, K. Liu, A. K. Schmid, A. Fert, and M. Chshiev, Significant DzyaloshinskiiMoriya Interaction at Graphene-Ferromagnet Interfaces Due to the Rashba Effect, Nat. Mater. 17, 605 (2018).

[26] F. Ajejas, A. Gudin, R. Guerrero, A. A. Barcelona, J. M. Diez, L. D. Costa, P. Olleros, M. A. Nino, S. Pizzini, J. Vogel, M. Valvidares, P. Gargiani, M. Cabero, M. Varela, J. Camarero, R. Miranda, and P. Perna, Unraveling Dzyaloshinskii-Moriya Interaction and Chiral Nature of Graphene/Cobalt Interface, Nano Lett. 18, 5364 (2018).

[27] G. Chen, A. Mascaraque, H. Y. Jia, B. Zimmermann, M. Robertson, R. Lo Conte, M. Hoffmann, M. A. G. Barrio, H. F. Ding, R. Wiesendanger, E. G. Michel, S. Blügel, A. K. Schmid, and K. Liu, Large Dzyaloshinskii-Moriya 
Interaction Induced by Chemisorbed Oxygen on a Ferromagnet Surface, Sci. Adv. 6, eaba4924 (2020).

[28] H. Yang, A. Thiaville, S. Rohart, A. Fert, and M. Chshiev, Anatomy of Dzyaloshinskii-Moriya Interaction at $\mathrm{Co} / \mathrm{Pt}$ Interfaces, Phys. Rev. Lett. 115, 267210 (2015).

[29] H. Y. Jia, B. Zimmermann, G. Michalicek, G. Bihlmayer, and S. Blügel, Electric Dipole Moment as Descriptor for Interfacial Dzyaloshinskii-Moriya Interaction, Phys. Rev. Mater. 4, 024405 (2020).

[30] A. Belabbes, G. Bihlmayer, F. Bechstedt, S. Blügel, and A. Manchon, Hund's Rule-Driven Dzyaloshinskii-Moriya Interaction at 3d-5d Interfaces, Phys. Rev. Lett. 117, 247202 (2016).

[31] A. Belabbes, G. Bihlmayer, S. Blügel, and A. Manchon, Oxygen-Enabled Control of Dzyaloshinskii-Moriya Interaction in Ultra-Thin Magnetic Films, Sci. Rep. 6, 24634 (2016).

[32] B. Hjorvarsson, J. A. Dura, P. Isberg, T. Watanabe, T. J. Udovic, G. Andersson, and C. F. Majkrzak, Reversible Tuning of the Magnetic Exchange Coupling in $\mathrm{Fe} / \mathrm{V}$ (001) Superlattices Using Hydrogen, Phys. Rev. Lett. 79, 901 (1997).

[33] D. Sander, W. Pan, S. Ouazi, J. Kirschner, W. Meyer, M. Krause, S. Muller, L. Hammer, and K. Heinz, Reversible H-Induced Switching of the Magnetic Easy Axis in $\mathrm{Ni} / \mathrm{Cu}$ (001) Thin Films, Phys. Rev. Lett. 93, 247203 (2004).

[34] A. A. Khajetoorians, M. Valentyuk, M. Steinbrecher, T. Schlenk, A. Shick, J. Kolorenc, A. I. Lichtenstein, T. O. Wehling, R. Wiesendanger, and J. Wiebe, Tuning Emergent Magnetism in a Hund's Impurity, Nat. Nanotechnol. 10, 958 (2015).

[35] J. Y. Ni, P. S. Wang, J. L. Lu, and H. J. Xiang, Realizing Magnetoelectric Coupling with Hydrogen Intercalation, Phys. Rev. Lett. 122, 117601 (2019).

[36] P. J. Hsu, L. Rozsa, A. Finco, L. Schmidt, K. Palotas, E. Vedmedenko, L. Udvardi, L. Szunyogh, A. Kubetzka, $\mathrm{K}$. von Bergmann, and R. Wiesendanger, Inducing Skyrmions in Ultrathin Fe Films by Hydrogen Exposure, Nat. Commun. 9, 1571 (2018).

[37] B. Yang, Q. Cui, J. Liang, M. Chshiev, and H. Yang, Reversible Control of Dzyaloshinskii-Moriya Interaction at the Graphene/Co Interface via Hydrogen Absorption, Phys. Rev. B 101, 014406 (2020).

[38] B. Santos, S. Gallego, A. Mascaraque, K. F. McCarty, A. Quesada, A. T. N'Diaye, A. K. Schmid, and J. de la Figuera, Hydrogen-Induced Reversible Spin-Reorientation Transition and Magnetic Stripe Domain Phase in Bilayer Co on $R u(0001)$, Phys. Rev. B 85, 134409 (2012).

[39] Q. Dubout, F. Donati, C. Wackerlin, F. Calleja, M. Etzkorn, A. Lehnert, L. Claude, P. Gambardella, and H. Brune, Controlling the Spin of Co Atoms on Pt(111) by Hydrogen Adsorption, Phys. Rev. Lett. 114, 106807 (2015).

[40] A. J. Tan, M. T. Huang, C. O. Avci, F. Buttner, M. Mann, W. $\mathrm{Hu}$, C. Mazzoli, S. Wilkins, H. L. Tuller, and G. S. D. Beach, Magneto-Ionic Control of Magnetism Using a Solid-State Proton Pump, Nat. Mater. 18, 35 (2019).

[41] U. Bauer, L. Yao, A. J. Tan, P. Agrawal, S. Emori, H. L. Tuller, S. van Dijken, and G. S. D. Beach, Magneto-Ionic Control of Interfacial Magnetism, Nat. Mater. 14, 174 (2015).
[42] C. Bi, Y. Liu, T. Newhouse-Illige, M. Xu, M. Rosales, J. W. Freeland, O. Mryasov, S. Zhang, S. G. E. te Velthuis, and W. G. Wang, Reversible Control of Co Magnetism by Voltage-Induced Oxidation, Phys. Rev. Lett. 113, 267202 (2014).

[43] D. A. Gilbert, J. Olamit, R. K. Dumas, B. J. Kirby, A. J. Grutter, B. B. Maranville, E. Arenholz, J. A. Borchers, and K. Liu, Controllable Positive Exchange Bias via Redox-Driven Oxygen Migration, Nat. Commun. 7, 11050 (2016).

[44] C. Leighton, Electrolyte-Based Ionic Control of Functional Oxides, Nat. Mater. 18, 13 (2019).

[45] C. Navarro-Senent, A. Quintana, E. Menéndez, E. Pellicer, and J. Sort, Electrolyte-Gated Magnetoelectric Actuation: Phenomenology, Materials, Mechanisms, and Prospective Applications, APL Mater. 7, 030701 (2019).

[46] P. D. Murray, D. A. Gilbert, A. J. Grutter, B. J. Kirby, D. Hernandez-Maldonado, M. Varela, Z. E. Brubaker, W. Liyanage, R. V. Chopdekar, V. Taufour, R. J. Zieve, J. R. Jeffries, E. Arenholz, Y. Takamura, J. A. Borchers, and K. Liu, Interfacial-Redox-Induced Tuning of Superconductivity in $\mathrm{YBa}_{2} \mathrm{Cu}_{3} \mathrm{O}_{7-d}$, ACS Appl. Mater. Interfaces 12, 4741 (2020).

[47] I. Chorkendorff, J. N. Russell, and J. T. Yates, Hydrogen Implantation in Ni(111)—A Study of $\mathrm{H}_{2}$ Desorption Dynamics from the Bulk, Surf. Sci. 182, 375 (1987).

[48] K. Christmann, Interaction of Hydrogen with SolidSurfaces, Surf. Sci. Rep. 9, 1 (1988).

[49] B. Bhatia and D. S. Sholl, Chemisorption and Diffusion of Hydrogen on Surface and Subsurface Sites of Flat and Stepped Nickel Surfaces, J. Chem. Phys. 122, 204707 (2005).

[50] J. Greeley and M. Mavrikakis, Surface and Subsurface Hydrogen: Adsorption Properties on Transition Metals and Near-Surface Alloys, J. Phys. Chem. B 109, 3460 (2005).

[51] K. Christmann, R. J. Behm, G. Ertl, M. A. Van Hove, and W. H. Weinberg, Chemisorption Geometry of Hydrogen on Ni(111): Order and Disorder, J. Chem. Phys. 70, 4168 (1979).

[52] A. L. F. Cauduro, L. H. Hess, D. F. Ogletree, J. W. Schwede, and A. K. Schmid, Tailoring Low Energy Electron Absorption via Surface Nano-Engineering of Cesiated Chromium Films, Appl. Phys. Lett. 115, 071602 (2019).

[53] K. Christmann, O. Schober, G. Ertl, and M. Neumann, Adsorption of Hydrogen on Nickel Single Crystal Surfaces, J. Chem. Phys. 60, 4528 (1974).

[54] J. N. Russell, I. Chorkendorff, A. M. Lanzillotto, M. D. Alvey, and J. T. Yates, Angular Distributions of $\mathrm{H}_{2}$ Thermal Desorption: Coverage Dependence on Ni(111), J. Chem. Phys. 85, 6186 (1986).

[55] Z. Huesges and K. Christmann, Interaction of Hydrogen with a Cobalt(0001) Surface, Z. Phys. Chem. 227, 881 (2013).

[56] See Supplemental Material at http://link.aps.org/ supplemental/10.1103/PhysRevX.11.021015 for supplemental figures, sample preparation, and time-dependent work-function measurements.

[57] J. Greeley, T. F. Jaramillo, J. Bonde, I. Chorkendorff, and J. K. Nørskov, Computational High-Throughput Screening of Electrocatalytic Materials for Hydrogen Evolution, Nat. Mater. 5, 909 (2006). 
[58] G. Chen, T. Ma, A. T. N'Diaye, H. Kwon, C. Won, Y. Wu, and A. K. Schmid, Tailoring the Chirality of Magnetic Domain Walls by Interface Engineering, Nat. Commun. 4, 2671 (2013).

[59] S. J. Jenkins and S. J. Pratt, Beyond the Surface Atlas: A Roadmap and Gazetteer for Surface Symmetry and Structure, Surf. Sci. Rep. 62, 373 (2007).

[60] K. H. Ernst, Molecular Chirality at Surfaces, Phys. Status Solidi B 249, 2057 (2012).

[61] B. Schweflinghaus, B. Zimmermann, M. Heide, G. Bihlmayer, and S. Blügel, Role of Dzyaloshinskii-Moriya Interaction for Magnetism in Transition-Metal Chains at Pt Step Edges, Phys. Rev. B 94, 024403 (2016).

[62] G. Chen, A. T. N'Diaye, S. P. Kang, H. Y. Kwon, C. Won, Y. Wu, Z. Q. Qiu, and A. K. Schmid, Unlocking Bloch-Type Chirality in Ultrathin Magnets through Uniaxial Strain, Nat. Commun. 6, 6598 (2015).

[63] A. Fert and P. M. Levy, Role of Anisotropic Exchange Interactions in Determining the Properties of Spin-Glasses, Phys. Rev. Lett. 44, 1538 (1980).
[64] Y. K. Park, D. Y. Kim, J. S. Kim, Y. S. Nam, M. H. Park, H. C. Choi, B. C. Min, and S. B. Choe, Experimental Observation of the Correlation between the Interfacial DzyaloshinskiiMoriya Interaction and Work Function in Metallic Magnetic Trilayers, NPG Asia Mater. 10, 995 (2018).

[65] S. Parkin and S.-H. Yang, Memory on the Racetrack, Nat. Nanotechnol. 10, 195 (2015).

[66] E. C. Burks, D. A. Gilbert, P. D. Murray, C. Flores, T. E. Felter, S. Charnvanichborikarn, S. O. Kucheyev, J. D. Colvin, G. Yin, and K. Liu, 3D Nanomagnetism in Low Density Interconnected Nanowire Networks, Nano Lett. 21, 716 (2021).

[67] U. Bauer, S. Emori, and G. S. D. Beach, Voltage-Controlled Domain Wall Traps in Ferromagnetic Nanowires, Nat. Nanotechnol. 8, 411 (2013).

[68] T. O. Menteş, A. Locatelli, L. Aballe, and E. Bauer, Stress Induced Stripe Formation in Pd/W(110), Phys. Rev. Lett. 101, 085701 (2008).

[69] R.-Q. Jia and H. Zhao, A Fast Algorithm for the Total Variation Model of Image Denoising, Adv. Comput. Math. 33, 231 (2010). 